\title{
Versatile fusion source integrator AFSI for fast ion and neutron studies in fusion devices
}

\author{
Paula Sirén ${ }^{1}$, Jari Varje ${ }^{2}$, Simppa Äkäslompolo ${ }^{2, *}$, Otto Asunta ${ }^{2,3}$, Carine Giroud ${ }^{4}$, \\ Taina Kurki-Suonio ${ }^{2}$, Henri Weisen ${ }^{5}$ and the JET Contributors ${ }^{\dagger}$ \\ ${ }^{1}$ VTT Technical Research Centre of Finland, P.O Box 1000, 02044 VTT, Finland \\ ${ }^{2}$ Department of Applied Physics, Aalto University, FI-00076 AALTO, Finland \\ ${ }^{3}$ Tokamak Energy Ltd, 120A Olympic Avenue, Milton Park, Abingdon, OX14 4SA, UK \\ ${ }^{4}$ CCFE, Culham Science Centre, Abingdon, Oxon, OX14 3DB, UK \\ ${ }^{5}$ Swiss Plasma Center, EPFL, CH-1015 Lausanne, Switzerland
}

August 24, 2017

\begin{abstract}
ASCOT Fusion Source Integrator AFSI, an efficient tool for calculating fusion reaction rates and characterizing the fusion products, based on arbitrary reactant distributions, has been developed and is reported in this paper. Calculation of reactorrelevant D-D, D-T and D- ${ }^{3}$ He fusion reactions has been implemented based on the Bosch-Hale fusion cross sections. The reactions can be calculated between arbitrary particle populations, including Maxwellian thermal particles and minority energetic particles. Reaction rate profiles, energy spectra and full 4D phase space distributions can be calculated for the non-isotropic reaction products. The code is especially suitable for integrated modelling in self-consistent plasma physics simulations as well as in the Serpent neutronics calculation chain. Validation of the model has been performed for neutron measurements at the JET tokamak and the code has been applied to predictive simulations in ITER.
\end{abstract}

\footnotetext{
* Current address: Max-Planck-Institut für Plasmaphysik, Wendelsteinstr. 1, 17491 Greifswald, Germany

† See the author list of "Overview of the JET results in support to ITER" by X. Litaudon et al. to be published in Nuclear Fusion Special issue: overview and summary reports from the 26th Fusion Energy Conference (Kyoto, Japan, 1722 October 2016)
}

\section{Introduction}

Any energy production mechanism based on nuclear fusion or fission reactions inevitably involves neutrons and high energy gamma photons. Both induce further nuclear reactions in the materials surrounding the reactor and, thus, their production profiles as well as transport in material structures has to be well understood. In traditional fission power, this neutron transport in materials is a widely studied topic with highly developed tools. On the other hand, in fusion-based devices, the connection between plasma fuel and neutronics has not been considered in detail. Typically, simple analytic approximations [1] have been used. However, the same methods are applicable to fusion reactors and current experimental devices with small modifications to the neutron source and the geometry.

To study neutronics in fusion devices, the prerequisite for reliable simulations is a detailed and realistic neutron source. In a full-scale reactor, the source can be assumed to consist almost entirely of thermonuclear reactions in the plasma fuel, and thus it can be readily evaluated even analytically based on a Maxwellian velocity distribution. However, in today's devices, the fusion production is dominated by externally produced fast ion population reacting with the thermal plasma. Such energetic ions are typically produced by either neutral beam injection or radio frequency heating. Only 
very crude analytical forms can be assumed for such population, so a need for a more refined way of evaluating the neutron source rates is pressing.

A realistic fusion source is equally important for studying the charged particles produced in fusion. Because they are confined by the magnetic field, they deposit their energy in the plasma, heating up the fuel and maintaining sufficient conditions for fusion. The large amount of energetic fusion products in reactors also affects the plasma equilibrium and stability, which must be included in any reactor control systems. Additionally, particles that escape the confinement can impact the vessel walls and cause localised heating and damage. These losses can be diagnosed using various detectors that measure the velocity space distribution of the lost particles. Interpreting these results requires a source model that takes into account not only the production rate but also the energy spectrum and even non-isotropic velocity distribution of the fusion products.

Fusion products, including those due to fast ion reactions, are calculated by several fast-ion codes such as TRANSP/NUBEAM [2] as well as dedicated fusion product codes such as GENESIS $[3,4,5]$ and DRESS [6]. In this contribution we present ASCOT Fusion Source Integrator AFSI, a tool for obtaining realistic fusion production rates and spectra for thermonuclear and fast-ion induced fusion reactions. The fast ions can be given as arbitrary 4D distribution functions from codes such as the orbit-following code ASCOT [7]. AFSI can then be used to calculate the fusion products either with fast analytic or semi-analytic models or with a versatile but heavier Monte Carlo method. These alternative models make the code especially suitable for integrated modelling, where rapid fusion product calculations are needed, but full velocity space description of the products can also be obtained.

The structure of this paper is as follows: First, in section 2, the mathematical models for calculating fusion product distributions are presented. These consist of separate approximations for thermal particle reactions, thermal-fast reactions and fast particle reactions. The code implementation, including required input and output data as well as coupled modelling with integrated codes are described in section 3. Benchmarks and validation with data from the JET tokamak are presented in section 4. Finally, the models and results are summarized and applications are discussed in section 5 .

\section{Mathematical description}

Fusion production rates in fusion plasmas can generally be simplified to two spatial dimensions due to approximate axisymmetry in the toroidal direction. They can be expressed in the poloidal plane as a function of $(\mathrm{R}, \mathrm{z})$ coordinates, where $\mathrm{R}$ is the major radius of the torus, or in $(\rho, \theta)$ coordinates, where $\theta$ is the poloidal angle and $\rho$ is a normalised radial coordinate defined on the concentric magnetic flux surfaces as $\rho=\sqrt{\left(\psi-\psi_{\text {axis }}\right) /\left(\psi_{\text {sep }}-\psi_{\text {axis }}\right)}$, where $\psi$ is the poloidal flux and $\psi_{\text {axis }}$ and $\psi_{\text {sep }}$ are its values at the magnetic axis and separatrix. This can further be approximated by a $1.5 \mathrm{D}$ approach, where the fusion production is averaged over the poloidal angle, taking into account the geometrical factors through the plasma elongation, triangularity and aspect ratio. This approach is particularly useful for thermonuclear fusion, where the reactant temperature and density can be assumed to be constant on magnetic flux surfaces.

The $3 \mathrm{D}$ velocity space distribution can likewise be simplified due to the motion of the particles along the magnetic field lines. Averaging over the rapid gyro motion of the particles around the field lines, their velocity can be divided into components parallel $v_{\|}$and perpendicular $v_{\perp}$ to the magnetic field. Alternatively, the velocity space coordinates can be expressed by the particle energy $E$ and pitch $\xi=v_{\|} /|\vec{v}|$.

AFSI includes three models for the fusion product distribution: 1) Analytic model, 2) Semianalytic model and 3) Monte Carlo model. The first model is a simple analytic formula for thermonuclear fusion, where both reactants originate in the Maxwellian thermal plasma. The second model uses a simulated fast particle population interacting with the thermal plasma, such as in the case of beam-thermal fusion. The third, most recently developed model calculates fusion products from arbitrary reactant distributions, which can represent either thermal or fast particles, such as in the case of beam-beam fusion. This model also includes calculation of a non-Maxwellian product energy spectrum. 


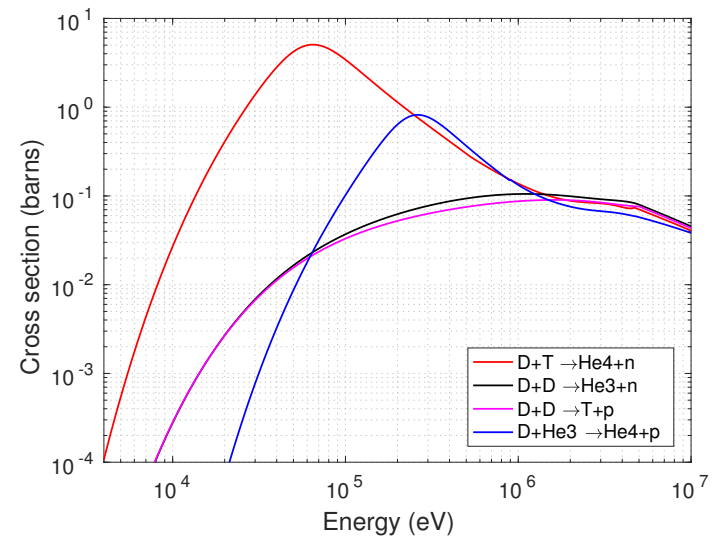

Figure 1: Fusion cross sections for DD, DT and DHe3 fusion as a function of the center-of-mass energy according to Bosch-Hale [8].

All models use the Bosch-Hale parametrisation [8] for the fusion cross sections (Figure 1) for the typical fusion reactions between deuterium, tritium and helium

$$
\begin{gathered}
D+D \rightarrow{ }^{3} \mathrm{He}+n \\
D+D \rightarrow T+p \\
D+T \rightarrow{ }^{4} \mathrm{He}+n \\
D+{ }^{3} \mathrm{He} \rightarrow{ }^{4} \mathrm{He}+p .
\end{gathered}
$$

The thermal model uses the rate coefficients $\langle\sigma v\rangle$ averaged over Maxwellian reactant populations, while the two other models use the cross sections $\sigma$ directly, both from [8].

\section{Analytic model}

The thermonuclear fusion products are computed in two steps. First, the reactivity between two Maxwellian ion species $i$ and $j$, with densities $n_{i}$ and $n_{j}$ is calculated simply as

$$
R_{\mathrm{Th}}=\frac{1}{1+\delta_{i j}} n_{i} n_{j}\langle\sigma v\rangle
$$

where $\delta_{i j}$ is the Kronecker delta function (to avoid double counting reactions from the same population) and $\langle\sigma v\rangle$ is the rate coefficient averaged over two Maxwellian velocity distributions, evaluated using the parametrisation in [8]. The distributions, and thus temperatures, are assumed to be identical for all ion species, which is a reasonable assumption for a stationary plasma. For identical ion species, the reaction rate is divided by 2 to avoid double counting reactions from identical particles.

After this, the energy spectrum of the fusion products is determined from an analytic expression based on the Maxwellian distribution of the source particles [9]

$f\left(E_{n}\right) d E_{n}=d E_{n} \exp \left[-\left(E_{n}-\left\langle E_{n}\right\rangle\right)^{2} / \frac{4 m_{n} T\left\langle E_{n}\right\rangle}{m_{n}+m_{a}}\right]$

where $E_{n}$ and $m_{n}$ are the energy and mass of the product particle, $\left\langle E_{n}\right\rangle$ is the mean energy of the product, $T$ is the ion temperature and $m_{a}$ is the mass of the second fusion product. This results in a Gaussian energy distribution for the fusion products, which is then normalised using the fusion rate calculated in the first step to get the distribution of the thermonuclear fusion products.

\section{Semi-analytic model}

The reaction rates between a fast ion population and the thermal plasma are calculated from the interaction between a Maxwellian $\left(f_{T}\left(\vec{v}_{T}\right)\right)$ and an arbitrary ion distribution such as the NBI slowingdown distribution $\left(f_{B}\left(\vec{v}_{B}\right)\right)$. Using the relative velocity $\vec{u}=\vec{v}_{T}-\vec{v}_{B}$, the reactivity can be expressed as the integral

$$
\begin{aligned}
R_{\mathrm{Th}-\mathrm{F}}= & \iiint_{\vec{v}_{\mathrm{B}}} \iiint_{\vec{v}_{\mathrm{T}}} f_{\mathrm{T}}\left(\vec{v}_{\mathrm{T}}\right) \sigma\left(\left|\vec{v}_{\mathrm{B}}-\vec{v}_{\mathrm{T}}\right|\right) \\
& f_{\mathrm{B}}\left(\vec{v}_{\mathrm{B}}\right)\left|\vec{v}_{\mathrm{B}}-\vec{v}_{\mathrm{T}}\right| \mathrm{d} \vec{v}_{\mathrm{T}} \mathrm{d} \vec{v}_{\mathrm{B}} \\
= & \iiint_{\vec{v}_{\mathrm{B}}} \underbrace{\iiint_{\vec{u}} f_{\mathrm{T}}\left(\vec{u}+\vec{v}_{\mathrm{B}}\right) \sigma(|\vec{u}|)|\vec{u}| \mathrm{d} \vec{u}}_{\langle\sigma v\rangle_{\mathrm{BT}}\left(\left|\vec{v}_{\mathrm{B}}\right|, T, m_{\mathrm{T}}\right)} \\
& f_{\mathrm{B}}\left(\vec{v}_{\mathrm{B}}\right) \mathrm{d} \vec{v}_{\mathrm{B}}
\end{aligned}
$$

which thus is a function of the beam ion distribution function and a velocity-averaged rate coefficient $\langle\sigma v\rangle_{B T}$. Integrating over the Maxwellian distribution, the rate coefficient can be expressed as a function of the beam ion velocity and the temperature $T$ as

$$
\begin{aligned}
\langle\sigma v\rangle_{\mathrm{BT}}= & -\frac{\sqrt{m_{\mathrm{T}}}}{v_{\mathrm{B}} \sqrt{2 \pi T}} \int_{0}^{\infty} \sigma(u) u^{2} \\
& {\left[e^{\frac{-m_{\mathrm{T}}\left(u+v_{\mathrm{B}}\right)^{2}}{2 T}}-e^{\frac{-m_{\mathrm{T}}\left(u-v_{\mathrm{B}}\right)^{2}}{2 T}}\right] \mathrm{d} u(9) }
\end{aligned}
$$


By numerically integrating and tabulating the rate coefficients, this method can be used to rapidly calculate the beam-thermal reaction rate. However, this procedure does not yield the energy spectrum of the products.

\section{Monte Carlo model}

In the Monte Carlo model, the fusion product distribution is calculated by numerically integrating equation (7) for arbitrary reactant distributions $f_{B}$ and $f_{T}$, which can represent any particle population, such as different NBI species or RFaccelerated minorities. The integral is evaluated using the Monte Carlo method of sampling pairs of reactant particles from a $(\mathrm{R}, \mathrm{z}, \xi, \mathrm{E})$ or $\left(\mathrm{R}, \mathrm{z}, \mathrm{v}_{\|}, \mathrm{v}_{\perp}\right)$ distribution function and calculating the resulting fusion production rate. The velocity space distribution of the products is simultaneously collected in a $2 \mathrm{D}$ histogram for each grid point.

The reactant particle pairs are sampled from the given $4 \mathrm{D}$ distribution functions. The velocities of the particles are represented in center of mass coordinates aligned with the magnetic field. The perpendicular velocity components are sampled from a uniform distribution representing the averaging over the helical gyro motion around the field line. The product particle velocity components are then calculated from the energy and momentum conservation laws in the center of mass coordinates. For example, in the case of a DT reaction, with reactants with masses $m_{1}$ and $m_{2}$ and products with masses $m_{N}$ and $m_{A}$ these give

$$
\begin{array}{r}
E_{\mathrm{tot}}=\frac{1}{2} m_{1}\left|\vec{v}_{1, \mathrm{CM}}\right|^{2}+\frac{1}{2} m_{2}\left|\vec{v}_{2, \mathrm{CM}}\right|^{2}+Q \\
=\frac{1}{2} m_{N}\left|\vec{v}_{\mathrm{N}, \mathrm{CM}}\right|^{2}+\frac{1}{2} m_{A}\left|\vec{v}_{\mathrm{A}, \mathrm{CM}}\right|^{2} \\
m_{1} \vec{v}_{1, \mathrm{CM}}+m_{2} \vec{v}_{2, \mathrm{CM}}=m_{N} \vec{v}_{\mathrm{N}, \mathrm{CM}}+m_{A} \vec{v}_{\mathrm{A}, \mathrm{CM}}
\end{array}
$$

where $Q$ is the reaction energy (such as $17.1 \mathrm{MeV}$ for DT fusion) and $\vec{v}_{1, \mathrm{CM}}, \vec{v}_{2, \mathrm{CM}} \vec{v}_{\mathrm{N}, \mathrm{CM}}$ and $\vec{v}_{\mathrm{A}, \mathrm{CM}}$ are the center of mass velocities of the reactants and the products. For defining the product velocities, the unit velocity vector $\hat{v}_{N}$ is sampled uniformly on the unit sphere, and the product velocity is calculated as

$$
\vec{v}_{\mathrm{N}, \mathrm{CM}}=\sqrt{\frac{2 E_{\mathrm{tot}}}{m_{N}\left(1+\frac{m_{N}}{m_{A}}\right)}} \hat{v}_{N}
$$

The product velocities are then transformed into laboratory coordinates. By collecting the velocity space coordinates for a sufficient sample population of reactant pairs, the energy spectrum and pitch distribution are obtained.

\section{Implementation and applications}

The three models described in the previous section make AFSI a flexible tool for calculating fusion production rates for all typical reactions in fusion plasmas. Depending on the fusion device and plasma scenario, not all of these reactions may be relevant. Thus by choosing appropriate models for a given application, these calculations can be performed rapidly. This enables the use of AFSI in integrated modelling with other plasma codes.

\section{Model implementation}

Figure 2 describes the required input data and types of output data that AFSI can calculate with the models described in section 2 . The input reactant particles can be given either as radial profiles for the thermal plasma population, or $4 \mathrm{D}$ distribution functions for fast ion species. Additionally, radial profiles can be converted into $4 \mathrm{D}$ distributions by sampling a Maxwellian distribution at the temperature specified by the profiles.

Based on the given inputs, AFSI can identify the reactant species and calculate fusion production rates and spectra for all combinations of reactant pairs for reaction types, including thermonuclear and, for example, beam-thermal and beam-beam fusion. The models used are chosen based on the input data such that the faster analytic and semianalytic models are preferred. However, if energy spectra or pitch distributions for the products are needed, the Monte Carlo model can be used for all reactions.

The reaction rates are calculated in a $2 \mathrm{D}$ grid in the poloidal plane for each reactant and reaction type. The reaction rates can also be given as flux surface averaged radial profiles. The velocity space distribution by the Monte Carlo model is calculated for each grid point, resulting in 4D fusion product distributions. Finally, these distributions can be sampled for markers to be used in further test-particle orbit-following simulations. 




Figure 2: The three different fusion source models in AFSI and the associated input and output data.

\section{Code integration and applications}

In integrated plasma modelling, AFSI is used as a fusion product source for transport and heating \& current drive simulation codes. AFSI is given profiles and distribution functions representing the plasma and fast ion distributions, and it calculates fusion production rates either for 1.5D transport or slowing-down simulations as needed.

The code has been implemented as an actor in the ITM framework [10]. In the workflows, the code is supplied with CPO (Consistent Physical Object) data structures containing the magnetic geometry, plasma profiles and fast ion distributions from other actors. Fusion rates are then given either as radial profiles or as markers back to Monte Carlo actors such as the orbit-following code ASCOT. AFSI is being similarly integrated with the JINTRAC suite [11] at JET for rapid interpretive and predictive simulations.

AFSI can be used directly as a fusion product source for the Monte Carlo orbit-following code ASCOT, allowing fast-ion slowing-down and transport simulations. AFSI has already been applied to studies of alpha particle losses in ITER [12, 13], where the realistic temperature-dependent alpha source enabled the study of heat loads on the first wall due to fast ion losses.

Finally, AFSI can be used as a fusion neutron source for neutronics codes and synthetic diagnostics. AFSI has been coupled with the Monte Carlo neutron transport code Serpent $[14,15]$, where the realistic non-Maxwellian product spectrum enables the study of material effects due to high-energy neutrons. AFSI has also been applied as the source for ideal synthetic diagnostics corresponding to the JET neutron camera, for which neutron rates along multiple lines of sight were compared, as well as for the JET time-of-flight spectrometer [16].

\section{Validation and first results}

AFSI has previously been benchmarked with TRANSP-NUBEAM simulations in fusion product simulations in the ASDEX Upgrade tokamak, where calculated DD fusion rates were found to closely agree with the TRANSP results [17]. In this chapter, further validation results are presented.

In the JET tokamak, total neutron production is measured with ${ }^{235} \mathrm{U}$ and ${ }^{238} \mathrm{U}$ fission chambers [19]. The first step of successful validation is to compare AFSI results with calibrated neutron rate measurements, which was performed for the JET discharge \#92436. The discharge is a high-performance baseline DD pulse with a central density of $8 \cdot 10^{19} 1 / \mathrm{m}^{3}$ and ion temperature of $9 \mathrm{keV}$. In this discharge, 28 $\mathrm{MW}$ of NBI and $5 \mathrm{MW}$ of 2 nd harmonic deuterium ICRH heating were used in the discharge. For the simulations, electron density and temperature profiles based on LIDAR, and ion temperature profiles based on charge exchange spectroscopy measurements were used, shown in Figure 3. Based on visible Bremsstrahlung measurements, $Z_{\text {eff }}$ in the dis- 


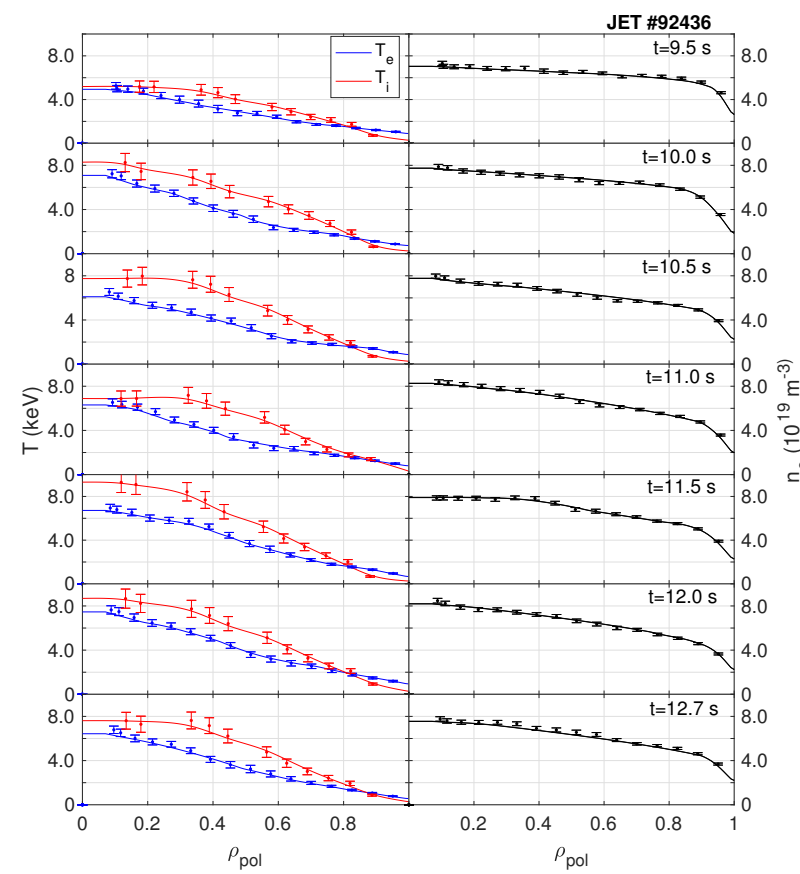

Figure 3: Plasma electron and ion temperature (left) and density profiles (right) from JET pulse \#92436.

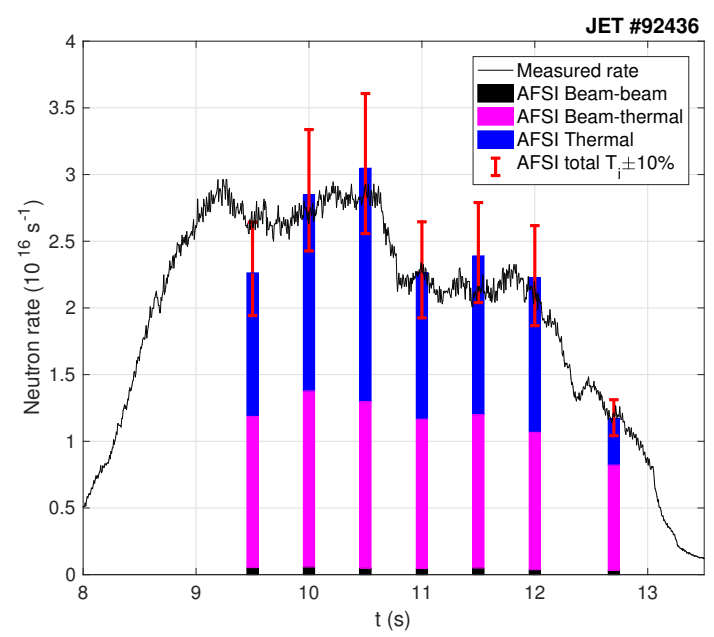

Figure 4: Fusion neutron production rates for thermal, beam-thermal and beam-beam reactions calculated by AFSI and compared with JET fission chamber measurements from the pulse \#92436. The error bars represent an uncertainty of $10 \%$ in ion temperature profiles. charge was approximately 1.8, which, was assumed to consists primarily of heavy impurities, resulting in low dilution. The slowing-down distribution for the beam ions was calculated using the orbitfollowing code ASCOT [7], with the beam ionization calculated using BBNBI [18].

AFSI simulation results for the total neutron rate were found to be consistent with the neutron rates based on the measurements of the fission chambers as shown in Figure 4. The difference between the total AFSI neutron rate and the experimental results is smaller than $20 \%$ for all simulated time slices. The contribution from beam-thermal particle reactions was the most significant, as expected due to the high NBI power. However, thermonuclear fusion contributed $30-60 \%$ of the total neutron rate due to the high ion temperature in the selected discharge, while beam-beam reactions produced approximately $1-2 \%$ of the neutrons. Possible contribution due to ICRH-thermal reactions was not taken into account in the simulation. However, the fraction of RF power compared to NBI heating in the discharge was low, resulting in only a small contribution from the ICRH-accelerated fast ions.

The calculated production rates include implicitly the uncertainty of the ion temperature measurements. As a sensitivity scan, an uncertainty of $10 \%$ in ion temperature measurements was assumed. This resulted in an uncertainty of up to $18 \%$ in the simulated neutron rates. This is due to the high temperature dependence of the fusion cross sections shown in Figure 1. Considering this uncertainty, as well as the uncertainty in the fission chamber measurements which is no more than $10 \%$ [19], the AFSI results are in good agreement with the experimental data.

The different fusion source models in AFSI were compared by calculating thermonuclear fusion production rates with all three models, using a 4D distribution function for the Maxwellian and isotropic thermal plasma. The calculations were done for the ITER baseline DT plasma [20] (Figure 5). The results are shown in figure 6 , where the reaction rates have been plotted along vertical and horizontal cross sections through the plasma center. The reaction rates were found to closely agree with each other. As the three models are independent and based on different assumptions, this crosscomparison validates the consistency of the three source models. 


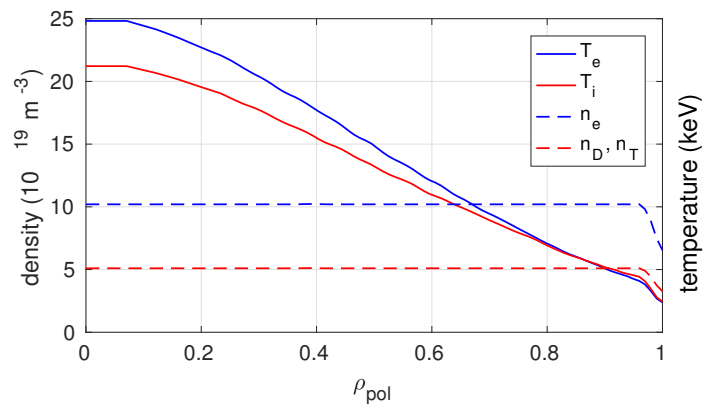

Figure 5: Density and temperature profiles in the ITER baseline scenario [20].

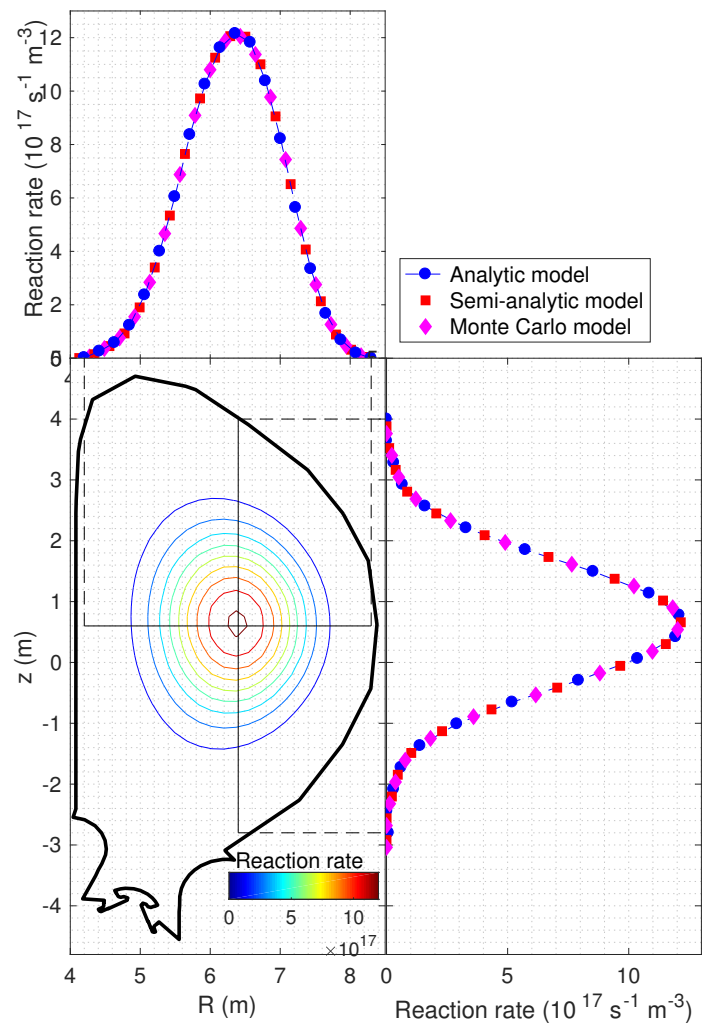

Figure 6: ITER baseline thermonuclear DT fusion production rates along the depicted vertical and horizontal lines calculated by the thermal, fastthermal and Monte Carlo models.

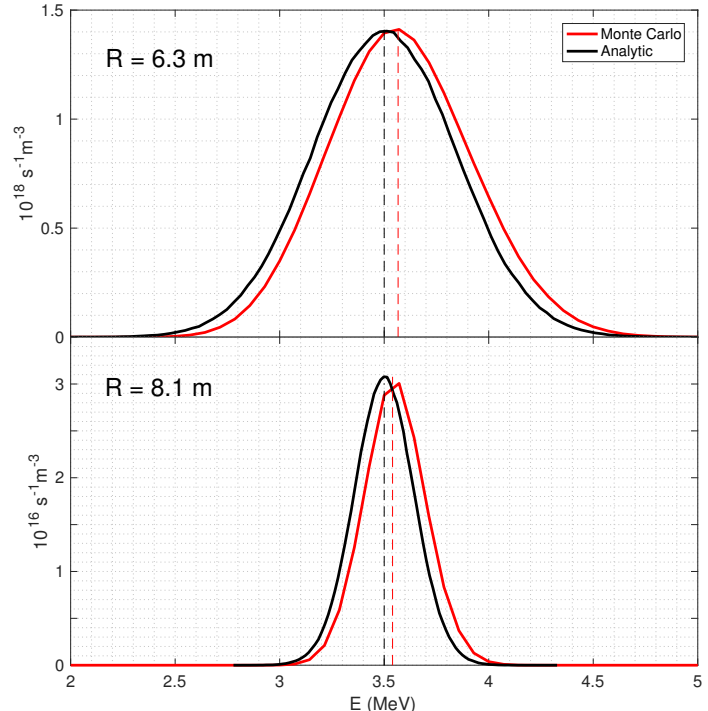

Figure 7: ITER baseline thermonuclear DT alpha particle spectra at two radial positions at the outer midplane corresponding to the core $(\mathrm{R}=6.3 \mathrm{~m})$ and the edge $(R=8.1 \mathrm{~m})$ calculated with the thermal and Monte Carlo models.

Also the thermonuclear alpha particle spectra were calculated for the ITER baseline scenario case using the Monte Carlo model with the Maxwellian 4D distributions, as shown in Figure 7. The results were compared with the analytical spectra obtained using the thermal model. Spectra were calculated at two radial positions near the core $(\mathrm{R}=6.3 \mathrm{~m})$ and the edge of the plasma $(\mathrm{R}=8.1 \mathrm{~m})$. The Monte Carlo model reproduces the shape of the analytical spectrum. Additionally, a temperature-dependent shift of $67 \mathrm{keV}$ in the core peak energy and $55 \mathrm{keV}$ in the edge peak energy is observed. This is due to the non-zero energy of the thermal particles, which is not taken into account by the thermal model, which assumes the nominal energy $\left\langle E_{\alpha}\right\rangle=3.5 \mathrm{MeV}$ for the alpha particles.

Finally, neutron spectra were calculated for the ITER baseline scenario for thermonuclear, beamthermal and beam reactions, presented in Figure 8. Due to the $1 \mathrm{MeV}$ negative-ion deuterium beams, the neutron energies reach up to $16.5 \mathrm{MeV}$ for the beam-thermal reactions between the beam ions and thermal tritium. This is an important consideration for activation analysis, where material-specific 


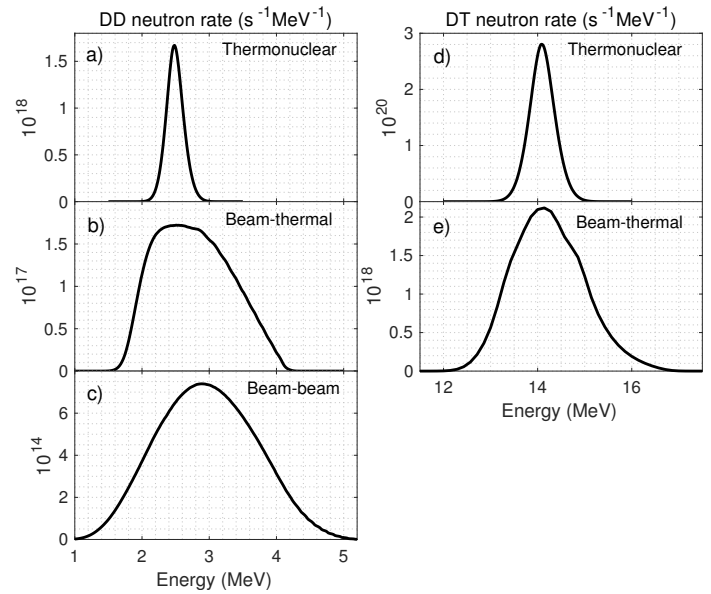

Figure 8: ITER baseline scenario neutron spectra for DD and DT thermonuclear, beam-thermal and beam-beam reactions.

threshold reactions may exist at energies higher than the nominal fusion neutron energy.

\section{Summary and future work}

In this paper, the fusion source models for thermal, fast-thermal and fast-fast particle reactions in the ASCOT Fusion Source Integrator AFSI have been presented. Additionally, the integration of the code with other code systems and as a part of an analysis chain in reactor-relevant applications has been described. Finally, quantitative validation with JET data and sample cases in predicted ITER plasmas have been presented. Neutron production calculated with AFSI was found to quantitatively agree with measured neutron rates, and the different models were shown to be consistent with each other when used to simulate the thermal fusion in the ITER baseline scenario.

In plasma physics simulations, the realistic temperature-dependent energy spectrum improves the study of alpha particle losses, as the energy affects the orbit width and consequent transport of the particles, as well as the energy of the lost particles. Further studies include considering the effect of the wider alpha energy spectrum due to beam-thermal and beam-beam reactions on the alpha losses and resulting non-uniform wall loads. Additionally, reactions due to fast ICRH accelerated ions and knock-on collisions with fusion al- pha particles will be studied. These will result in tail populations of $\mathrm{MeV}$-range fuel ions, leading in turn to tail population of fusion products with even higher energies than shown here for NBI beam ions.

For neutronics, due to the realistic geometry and energy spectrum of its Monte Carlo model, AFSI is a more versatile tool than the analytical models that are typically used [1]. As a neutron source, AFSI has been applied not only as neutron source in activation and nuclear safety analysis, but also as an ideal synthetic neutron diagnostic which can simulate neutron spectra. An AFSI-based detailed neutron source enables the study of the effects of a realistic velocity space distribution. These include activation due to high-energy neutrons, anisotropic heating in power plant process modelling as well as neutron transport and multiplication in tritium breeding blankets. AFSI will also be an ideal tool for sensitivity analysis due to plasma physics related effects, such as the dependence on plasma temperature or shape.

While synthetic neutron diagnostics have been used for qualitative comparisons to neutron cameras and spectrometers [21], modelling of detector related effects is required for quantitative validation. This will be done by coupling the neutron source to neutron transport analysis of the diagnostic geometry with the Serpent code. Additionally, inclusion of tritium-tritium fusion, gammaproducing reactions, such as $\alpha$-Be and differential cross-sections for anisotropic reactions will further extend the applications of AFSI as a source for synthetic diagnostics.

\section{Acknowledgements}

This work has been carried out within the framework of the EUROfusion Consortium and has received funding from the Euratom research and training programme 2014-2018 under grant agreement No 633053. The views and opinions expressed herein do not necessarily reflect those of the European Commission. The work was partly funded by Walter Ahlström foundation. Some of the calculations were performed using computer resources within the Aalto University School of Science 'Science-IT' project. 


\section{References}

[1] Fausser C et al. 2012 Tokamak D$\mathrm{T}$ neutron source models for different plasma physics confinement modes, Fusion Engineering and Design 87 787-792 http://dx.doi.org/10.1016/j.fusengdes.2012.02.025

[2] Pankin A et al. 2004 The tokamak Monte Carlo fast ion module NUBEAM in the National Transport Code Collaboration library, Computer Physics Communications 159 157-184 http://dx.doi.org/10.1016/j.cpc.2003.11.002

[3] Nocente M et al. 2012 Neutron and gammaray emission spectroscopy as fast ion diagnostics in fusion plasmas, Doctoral dissertation https://boa.unimib.it/handle/10281/28397

[4] Nocente M et al. 2013 Reaction analysis of neutron emission from $\mathrm{D}$ and $\mathrm{DT}$ plasmas with/without 3He, Nuclear Fusion 53053010 https://doi.org/10.1088/0029$5515 / 53 / 5 / 053010$

[5] Tardocchi M et al. 2011 Spectral Broadening of Characteristic $\gamma$-Ray Emission Peaks from ${ }^{12} \mathrm{C}\left({ }^{3} \mathrm{He}, \mathrm{p} \gamma\right){ }^{14} \mathrm{~N}$ Reactions in Fusion Plasmas, Physical Review Letters 107205002 https://doi.org/10.1103/PhysRevLett.107.205002

[6] Eriksson J, Conroy S, Andersson Sundén E, Hellesen C 2016 Calculating fusion neutron energy spectra from arbitrary reactant distributions, Computer Physics Communications $199 \quad 40-46$ http://dx.doi.org/10.1016/j.cpc.2015.10.010

[7] Hirvijoki $\mathrm{E}$ et al. 2014 ASCOT: solving the kinetic equation of minority particle species in tokamak plasmas, Computer Physics Communications 185 1310-1321 http://dx.doi.org/10.1016/j.cpc.2014.01.014

[8] Bosch H S, Hale G 1992 Improved formulas for fusion cross-sections and thermal reactivities, Nuclear Fusion 32611 https://doi.org/10.1088/0029-5515/32/4/I07

[9] Brysk H 1973 Fusion neutron energies and spectra, Plasma Physics 15 611 https://doi.org/10.1088/0032-1028/15/7/001
[10] Coster D et al. 2010 IEEE Trans. Plasma Sci. $38 \quad 2085$ https://doi.org/10.1109/TPS.2010.2056707

[11] Romanelli $M$ et al. 2014 JINTRAC: a system of codes for integrated simulation of tokamak scenarios, Plasma and Fusion Research 9 Special Issue 2 http://dx.doi.org/10.1585/pfr.9.3403023

[12] Kurki-Suonio T et al. 2016 Effect of the European design of TBMs on ITER wall loads due to fast ions in the baseline (15 MA), hybrid (12.5 MA), steady-state (9 MA) and half-field (7.5 MA) scenarios, Nuclear Fusion 56112024 https://doi.org/10.1088/0029$5515 / 56 / 11 / 112024$

[13] Äkäslompolo S et al. 2015 ITER fast ion confinement in the presence of the European test blanket module, Nuclear Fusion 55093010 https://doi.org/10.1088/0029$5515 / 55 / 9 / 093010$

[14] Leppänen J et al. 2015 The Serpent monte carlo code: Status, development and applications in 2013, Ann Nucl. Energy 84 55-62 http://dx.doi.org/10.1016/j.anucene.2014.08.024

[15] Sirén P, Leppänen J 2016 Expanding the use of Serpent 2 to Fusion Applications: Development of a Plasma Neutron Source, In proc. PHYSOR 2016, Sun Valley, ID, May 1-6 2016.

[16] Sirén P et al. 2017 Synthetic neutorn diagnostics (KN3/TOFOR) in JET based on AFSIASCOT simulations, To be submitted to Journal of Instrumentation, Proceedings of the 2nd European Conference on Plasma Diagnostics, Bordeaux, 18-21 April 2017.

[17] Äkäslompolo S et al. 2015 Journal of Instrumentation $10 \quad \mathrm{P} 10012$ https://doi.org/10.1088/17480221/10/10/P10012

[18] Asunta O et al. 2015 Modelling neutral beams in fusion devices: Beamlet-based model for fast particle simulations, Computer Physics Communications 188 33-46 http://dx.doi.org/10.1016/j.cpc.2014.10.024 
[19] Syme D B et al. 2012 Fusion yield measurements on JET and their calibration, Nuclear Engineering and Design 246 185-190 http://dx.doi.org/10.1016/j.nucengdes.2011.08.003

[20] Polevoi A, Kavin A 2002 ITER plasma data for Scenario 4 (during burn). ITER D 22KZM2 v1.0. https://user.iter.org/?uid=22KZM2

[21] Sirén P et al. 2017 Synthetic neutron camera and spectrometer in JET based on AFSIASCOT simulations, Journal of Instrumentation in press 